\title{
An evaluation of knowledge, attitude and perception about adverse drug reactions and pharmacovigilance among intern doctors in a medical college teaching hospital of Sangli
}

\author{
Santoshkumar A. Shetti*, R. P. Limaye \\ Department of Pharmacology, Bharati Vidyapeeth (Deemed to be University) Medical College and Hospital, Sangli, \\ Maharashtra, India
}

Received: 20 April 2021

Revised: 13 May 2021

Accepted: 14 May 2021

*Correspondence:

Dr. Santoshkumar A. Shetti,

Email: shetti989@gmail.com

Copyright: (c) the author(s), publisher and licensee Medip Academy. This is an open-access article distributed under the terms of the Creative Commons Attribution Non-Commercial License, which permits unrestricted non-commercial use, distribution, and reproduction in any medium, provided the original work is properly cited.

\begin{abstract}
Background: Underreporting of various adverse drug reactions (ADRs) by consultants is a common incurable problem. National pharmacovigilance program is one of the ongoing programs to monitor the adverse drug reactions $\&$ reporting at the earliest to the nearby AMCs. As medical interns are budding doctors, the focus of this study was to evaluate the knowledge, attitude and perception about ADRs and pharmacovigilance in them.

Methods: A cross section questionnaire-based study was conducted after approval by our institutional ethics committee pretested and validated questions consisting of 20 questions (knowledge, attitude, perception) were administrated to medical interns. The filled questionnaires were collected and analysed.

Results: In our study, medical interns have fair enough idea about ADR and pharmacovigilance. In knowledge domain they were aware of term ADRs (100\%), pharmacovigilance (72.6\%). In attitude domain majority of interns (80.6\%) known availability of ADR forms, compulsory of pharmacovigilance unit $(90.3 \%)$. In perception domain very poor response from interns, not even a single intern had reported any ADR filled form and they don't know meaning of rechallenge and de-challenge, very few interns (9.7\%) know how to manage the ADRs in emergency conditions.

Conclusions: Under reporting problem can be improved by doing more teaching activities at undergraduate level and intern's level including various workshops, CMEs, problem-based teaching of adverse reactions in their curriculum. These exercises will improve their reporting frequency and sensitize the interns from the undergraduate days itself in their upcoming clinical practice in community.
\end{abstract}

Keywords: Pharmacovigilance, Adverse drug reactions, Intern, Knowledge

\section{INTRODUCTION}

Fourth leading cause of death and treatment related morbidity is considered to be ADRs. ${ }^{1}$ Adverse drug reactions are defined as a 'one which is noxious and unintended and which occurs in doses normally used in human for prophylaxis, diagnosis or therapy of disease, or for the modification of physiological functions'. ${ }^{2}$ Similar way $\mathrm{WHO}$ has defined serious ADR 'untoward medical occurrence at any dose that results in death, life threatening, requires or prolongs hospitalization, or results in persistent or significant disability or incapacity'. ${ }^{3}$

Pharmacovigilance (PV) is defined as 'the science and activities relating to the detection, assessment, understanding and prevention of adverse effects or any other drug related problem'. WHO started its pharmacovigilance programme early in the 1960s. With the headquarters at Uppsala, the international activity for data collection in pharmacovigilance is going on. WHO 
promoted establishment of National PV centres in each country. The accumulated information is analysed to prepare reliable and balanced information to issue drug alerts. ${ }^{4}$

In India there is a strong belief that underreporting of ADRs is because of multiple reasons. Pharmacovigilance program of India (PvPI) was launched in India to enhance the voluntary reporting. In 2011 the national coordinating centre to monitor ADR shifted from AIIMS New Delhi to Indian Pharmacopoeia commission Ghaziabad. ${ }^{5}$

A new drug going through all tests like preclinical studies and then clinical trials, after entering in to the open market it is exposed to large patient population with variety of genetic makeup of people as compared to study subjects in clinical trials. Various factors like age, sex, genetic factors, food habit pattern, coexisting comorbidities, geographical variations and concomitant medications play a much significant role in occurrence of ADRs. Any health worker like doctors, nurse, dentist, can voluntarily report the ADRs. Active participation of health workers plays an important role in the successful implementation of pharmacovigilance programme.

Objectives of the study were to assess the level of knowledge, attitude in interns to assess the level of perception and attitude towards reporting of ADRs working in Bharati medical hospital, a tertiary care teaching hospital of Bharati Vidyapeeth Medical College, Sangli in Southwest Maharashtra.

\section{METHODS}

It was a cross sectional questionnaire-based study, consisting of 20 questions covering knowledge, attitude and perception components randomly mixed in a questionnaire. This study was conducted in Bharati hospital attached to Bharati Vidyapeeth Medical College, a tertiary care medical teaching hospital situated in Sangli, South Maharashtra. Before initiating the study, it was presented to Institutional Human Ethics Committee and approval was taken. Total duration of study was 5 months from February 2018 to June 2018. In order to achieve the objectives a questionnaire was designed and pretested. Based upon Saurabh et al, we prepared questions to asses knowledge, attitude, perception related with ADRs and $\mathrm{PV}$. We compiled the questionnaire and pretested it before using it in the study.

All questions were first analysed and screened in detail and pre-tested, validated by expert staff. Based on the feedback from them certain questions were modified \& reframed. After finalizing questions and obtaining approval from the IEC study was conducted.

\section{Inclusion criteria}

All medical interns who gave voluntarily informed consent and responded to all questions were included.

\section{Exclusion criteria}

Those interns who were not willing to participate in study, who had incomplete responses and who didn't return the questionnaire response sheet were excluded.

Those interns who gave consent were provided the filling response sheets and told to answer the questions mentioned, and return to us within 4 days. Only those who had answered all the questions were taken for our analysis. The questionnaire consists of 20 questions in which six questions were on knowledge based, seven questions were on attitude based and remaining seven questions were on perception based of ADR reporting and PV.

Each correct response was given a score of ' 1 ' and wrong answer was given ' 0 ' total maximal score was 20 . A convenient purposive sample of 150 was selected. After obtaining consent from the medical interns, response sheets were given to them with instructions to fill it and return within four days. A total of 150 interns were given response sheets, whereas final numbers included for analysis were 124, as 26 were excluded from the final analysis as 18 were incompletely filled and $8 \mathrm{did}$ not return the response sheet in spite of multiple reminders.

\section{Statistical analysis}

The results were entered and analysed question wise and their percentage, proportions and means are used for descriptive statistics with the help of Microsoft excel 2010 spread sheet software.

\section{RESULTS}

In our study 124 completed response sheets from medical interns were analysed after removing incomplete respondents and those who did not return the response sheets even after multiple reminder. All the answers are mentioned in terms of numbers, percentages and mean $\pm \mathrm{SD}$, for KAP questionnaire. In our study number of male participants $72(58 \%)$ and females 52 (42\%), average age of all interns was 24.4 years.

In our questionnaire out of 20, we have given 6 knowledge based, 7 attitude-based and remaining 6 on perception based randomly in entire response sheet. Among knowledge-based questions, question 1 was regarding awareness of the term adverse drug reactions which was correctly answered by $100 \%$ of medical interns. Question 2 was regarding awareness of the term pharmacovigilance which was correctly answered by $72.6 \%$ of medical interns. Question 3 was regarding medical department taking care of ADR and PV, it was correctly answered by $24.2 \%$ only.

Question 11 was regarding reporting of ADRs to AMC (ADR monitoring centre) by non-medicos, which showed that only $35.5 \%$ were aware about it. Question 14 was about ADR reporting submission following route or 
process, for which only $13 \%$ answered correctly. Question 17 was concerned with location of National Coordinating
Centre in India, for which only $1.7 \%$ were answered correctly (Table 1).

Table 1: Knowledge among medical interns about ADR reporting and pharmacovigilance.

\begin{tabular}{|llll|}
$\begin{array}{l}\text { Quest- } \\
\text { ionna- } \\
\text { ire no. }\end{array}$ & $\begin{array}{l}\text { Questions were asked for assessing knowledge } \\
(\mathbf{n}=\mathbf{1 5 0}, \text { out of which } \mathbf{1 2 4} \text { are taken in to consideration) }\end{array}$ & $\begin{array}{l}\text { Correct } \\
\text { response } \\
\mathbf{N}(\%)\end{array}$ & $\begin{array}{l}\text { Incorrect } \\
\text { response }\end{array}$ \\
$\mathbf{1 .}$ & Are you aware of the term adverse drug reactions? & $124(100)$ & 0 \\
\hline 2. & Are you aware of the term pharmacovigilance? & $90(72.6)$ & $34(27.4)$ \\
\hline 3. & $\begin{array}{l}\text { Which medical department will take care of ADR reporting and } \\
\text { pharmacovigilance? }\end{array}$ & $30(24.2)$ & $94(75.8)$ \\
\hline 11. & Can non-medicos report ADR to AMC (ADR Monitoring Centre)? & $44(35.5)$ & $80(64.5)$ \\
\hline 14. & ADR report submission follows which order? & $16(13)$ & $108(87)$ \\
\hline 17. & Where is National Coordinating Centre of Pharmacovigilance located in India? & $02(1.7)$ & $122(98.3)$ \\
\hline
\end{tabular}

Table 2: Attitude among medical interns about ADR reporting and pharmacovigilance.

\begin{tabular}{|llll|}
$\begin{array}{llll}\text { Quest- } \\
\text { ionna- } \\
\text { ire no. }\end{array}$ & Questions were asked for assessing attitude & $\begin{array}{l}\text { Correct } \\
\text { response } \\
\mathbf{N}(\%)\end{array}$ & $\begin{array}{l}\text { Incorrect } \\
\text { response } \\
\mathbf{N}(\%)\end{array}$ \\
\hline 4. & Who can report adverse drug reactions? & $28(22.6)$ & $96(77.4)$ \\
\hline 7. & Are you aware of the ADR forms available in our college and hospital? & $100(80.6)$ & $24(19.4)$ \\
\hline 9. & Is it compulsory to have pharmacovigilance unit in medical college? & $112(90.3)$ & $12(9.7)$ \\
\hline 12. & Whether ADR reporting should be made compulsory for all doctors? & $120(96.8)$ & $04(3.2)$ \\
\hline 15. & What is known by the term phase IV clinical trial? & $14(11.3)$ & $110(88.7)$ \\
\hline 19. & $\begin{array}{l}\text { Do you think ADR reporting exercises should be included in second year } \\
\text { MBBS pharmacology practical classes? }\end{array}$ & $116(93.5)$ & $08(6.5)$ \\
\hline 20. & $\begin{array}{l}\text { Do you think PvPI programme will improve the morbidity and mortality of } \\
\text { patients in clinical practice? }\end{array}$ & $120(96.8)$ & $04(3.2)$ \\
\hline
\end{tabular}

Table 3: Perception among medical interns about ADR reporting and pharmacovigilance.

\begin{tabular}{|c|c|c|c|}
\hline $\begin{array}{l}\text { Quest- } \\
\text { ionna- } \\
\text { ire no. }\end{array}$ & $\begin{array}{l}\text { Open ended question were asked for assessing perception towards ADR } \\
\text { and pharmacovigilance }\end{array}$ & $\begin{array}{l}\text { Correct } \\
\text { response } \\
\mathbf{N}(\%)\end{array}$ & $\begin{array}{l}\text { Incorrect } \\
\text { response } \\
\mathbf{N}(\%)\end{array}$ \\
\hline 5. & Where should you report? & $26(21)$ & $98(79)$ \\
\hline 6. & Which types of ADRs should be reported? & $122(98.3)$ & $02(1.7)$ \\
\hline 8. & $\begin{array}{l}\text { Are you aware of the "suspected adverse drug reaction" android app by NCC- } \\
\text { PvPI for reporting of ADRs on play store of your smart phone? }\end{array}$ & $04(3.2)$ & $120(96.8)$ \\
\hline 10. & $\begin{array}{l}\text { The nearest ADR Monitoring Centre (AMC) located at which medical } \\
\text { college? }\end{array}$ & $06(4.8)$ & $118(95.2)$ \\
\hline 13. & Have you reported any ADR report till now? & No $(0)$ & \\
\hline 16. & $\begin{array}{l}\text { Can you explain re-challenge or de-challenge in reference to adverse drug } \\
\text { reaction correctly? }\end{array}$ & No $(0)$ & \\
\hline 18. & $\begin{array}{l}\text { Do you know how to manage ADRs in emergency, from progressing to } \\
\text { mortality of patients? }\end{array}$ & $12(9.7)$ & $112(90.3)$ \\
\hline
\end{tabular}

Seven questions were regarding attitude-based questions in our response sheets. Question 4 was regarding reporting of ADRs, for which only $22.6 \%$ knew the correct answers. Question 7 was regarding awareness of the ADR forms available in our college and hospital, for which only $80.6 \%$ knew it about availability of forms. Question 9 was concerned with PV unit in our medical college, for which $90.3 \%$ interns responded to have it compulsory in a medical college. Question 12 was concerned with reporting of ADRs by all doctors mandatory or compulsory, for which $96.8 \%$ interns agreed to have it compulsory. Question 15 was concerned with phase IV clinical trial, for which only $11.3 \%$ interns answered it correctly. Question 19 was regarding inclusion of ADR reporting exercises in our second year MBBS practical classes, for which $93.5 \%$ of interns agreed to have it in our practical classes. Question 20 was regarding PvPI programme will improve morbidity and mortality in clinical practice, for which $96.8 \%$ interns answered it correctly (Table 2). 
Seven questions were regarding perception-based questions in our response sheets. Question 5 was regarding where you should report the ADRs, for which only $21 \%$ of interns knew it where to report. Question 6 was regarding types of ADRs to be reported, for which $98.3 \%$ of interns answered correctly. Question 8 was regarding the awareness of Suspected ADR android app by NCC-PvPI for reporting of ADRs on playstore of smartphones, for which only $3.2 \%$ were aware about it. Question 10 was regarding the nearest ADR monitoring centre location, for which only $4.8 \%$ interns answered it correctly. Question 13 and 16 were two open ended questions asking about reporting of ADR till now and can they explain the dechallenge and re-challenge test in reference to ADRs, for which they replied as none of them have reported any kind of ADRs till now \& they don't have any idea of dechallenge and re-challenge test. Question 18 was regarding management of ADRs in emergency from progressing to mortality of patients, for which only $9.7 \%$ interns answered it stating that they know to manage the ADRs in emergency from progressing it to mortality (Table 3 ).

\section{DISCUSSION}

Pharmacovigilance is defined as 'the science and activities relating to the detection, assessment, understanding and prevention of adverse events or any other drug related problem. ${ }^{7}$ All kind of ADRs do cause a kind of morbidity, financial burden (long hospital-stay) and even mortality on our health care system.

All drugs which have got a mechanism of action used for a particular clinical condition do have the adverse effects in one or the other way, only the severity varies from drug to drug. Even placebos used for a clinical condition do have some kind of adverse effects.

Nowadays spontaneous reporting of ADRs is an important work of a citizen of India under national pharmacovigilance which involves all health care workers like doctor, nurses, pharmacist $\&$ even patients.

Our study was conducted in medical interns posted in various wards of clinical department in a tertiary care hospital of Sangli. As interns are one of the frontline health care-worker in patient care, and are upcoming doctors in community it's their moral responsibility to encounter various adverse effects of drugs and note it down to PV or ADR monitoring centre. There are many studies conducted to assess knowledge of health care providers regarding pharmacovigilance, however very few studies involved the medical interns. ${ }^{8-10}$

In our study almost all students were aware of the term adverse drug reactions. Around $72.6 \%$ of interns were aware of the term pharmacovigilance which is quite satisfactory. Only $24.2 \%$ interns were aware that pharmacology department will take care of ADR reporting and pharmacovigilance. These results were comparable and par with other studies conducted by Thakuria et al and Korde et al. ${ }^{11,12}$

Regarding awareness of reporting of ADRs to AMC by non-medicos was answered correctly by only $35.5 \%$. The order of submission of ADRs from ward to pharmacology department \& then to nearby AMC in GMC miraj was only known by $13 \%$ of interns, rest of them were unaware of procedure after filling the ADR form. Only $1.7 \%$ of interns were aware of the location of the National Coordinating Centre of Pharmacovigilance i.e. Ghaziabad. Over all knowledge of interns regarding pharmacovigilance was good. These results were better compared to study conducted by Korte et al, in which majority of interns scored very less in knowledge domain. ${ }^{12}$

Concerning the attitude awareness among interns, only $22.6 \%$ of them were aware that all health care workers can report the ADRs unlike majority were under impression that only doctors can report it. The availability of ADR filling forms in our hospital for entering the data were known by $80.6 \%$ of interns. Majority of the interns $90.3 \%$ were correct in having a pharmacovigilance unit in our college and hospital. ADR reporting should be made compulsory for all doctors, for which $96.8 \%$ interns were in favour of it and answered correctly. Regarding phase IV clinical trials only $11.3 \%$ interns were aware of it and answered. Then later inclusion of ADR reporting exercises in second year MBBS pharmacology practical classes, for which $93.5 \%$ were in favour of its inclusion for betterment of the students. Lastly regarding the attitude of interns towards improvement of morbidity and mortality of patients in clinical practice by including PvPI programme, $96.8 \%$ interns were answered it correctly. So, attitude towards ADR reporting was comparable to study conducted by Gupta et al. ${ }^{13}$

Concerning the perception about ADR and PV, 21\% of interns were aware about where to report the filled ADR forms whereas, rest of them had no idea where to report. Almost $98.3 \%$ of interns answered correctly regarding the types of ADRs to be reported. Majority of interns $96.8 \%$ don't have the idea and usage of "Suspected ADR" android app by NCC-PvPI for reporting of ADRs on playstore of their smartphone. The nearest ADR monitoring centre (which is GMC Miraj in our case) is not known by $95.2 \%$ of interns. No intern has reported any kind of ADR forms till during our study. They don't have any idea about dechallenge \& re-challenge test performed in relation to adverse drug reactions. Then regarding management of ADRs in emergency, only $9.7 \%$ were aware of it and answered correctly.

In our study interns answered comparatively satisfactorily towards knowledge and attitude, where as in case of perception there is still lot much improvement required.

Similar studies were done by Agarwal et al, where participants had enough good knowledge and attitude but there was marked decrease in practice of reporting nature 
of forms. ${ }^{14}$ Interns in our study have not reported even a single case of ADR forms, reason being various accounting for under reporting of ADR forms. Underreporting is a matter of serious concern for PvPI as various new drugs are coming in to market along with older drugs, which would lead to masking of the reactions \& conditions leading in increasing the morbidity \& mortality of patients. ${ }^{15}$ Kalaiselvan et al, found that reporting by non-health care professionals was only $0.016 \%$ which is very very less as compared to the incidence of ADRs occurring. ${ }^{16}$

The majority of the factors responsible for under reporting by interns may be because of their workload, lack of interest in picking up and reporting, lack of time, lack of concern of future days if not reported in time, lack of compulsion from institute towards reporting all these are accounted in poor response of ADR filling forms. Similar responses were found in other published studies describing various hurdles in ADR detection and reporting. ${ }^{17-19}$

In our study, majority of the interns have suggested that there is need to teach the ADR and pharmacovigilance topic in detail in UG curriculum. To support this it is seen recently that ADR filling exercises for MBBS students in practical classes has been introduced by new CBME curriculum as per NMC norms in competency based approach, which will improve the compliance of detecting and reporting of ADR forms. It will improve the quality care in future budding doctors. Hope there will be much improvement in pharmacovigilance in coming days.

\section{Limitations}

Limitations of the study were- (a) responses of close ended questions given by interns were totally depends on honesty of subjects included in study; (b) our study was restricted to one centre only, as these findings should not be applied for wider population. Many more studies to be carried out in similar nature to support the data.

\section{CONCLUSION}

The results of our present study showed that interns had enough amount of knowledge and lenient attitude towards pharmacovigilance, however there is lack of reporting exercises which has to be taken seriously and implemented in interns to increase the underreporting of forms. Hence various educational activities in $\mathrm{UG}$ curriculum will enhance their awareness and ADR reporting culture among interns and help in their future clinical practice in community.

\section{ACKNOWLEDGEMENTS}

We would like to thank all the medical intern participants for their active voluntary participation. We are thankful to Bharati hospital staff, Deptartment of Pharmacology, Bharati Vidyapeeth Medical College and Hospital, Sangli for providing their valuable support and constant motivation in carrying out this work.

Funding: No funding sources

Conflict of interest: None declared

Ethical approval: The study was approved by the Institutional Ethics Committee

\section{REFERENCES}

1. Brown SD, Landry FJ. Recognizing, reporting and reducing adverse drug reactions. South Med J. 2001;94:370-3.

2. Sriram S, Ghasemi A, Ramasamy R, Devi M, Balasubramanian R, Ravi TK, et al. Prevalence of adverse drug reactions at a private tertiary care hospital in south India. J Res Med Sci. 2011;16(1):1625.

3. Prajapati K, Desai M, Shah S, Panchal J, Kapadia J, Dikshit R. An analysis of serious adverse drug reactions at a tertiary care teaching hospital. Perspect Clin Res. 2016;7(4):181-6.

4. WHO. 4. What is Pharmacovigilance?, 2019. Available at: https://www.who.int/medicines/areas/. Accessed on 3 June 2019.

5. Upadhyaya HB, Vora MB, Nagar JG, Patel PB. Knowledge, attitude and practices toward pharmacovigilance and adverse drug reactions in postgraduate students of Tertiary Care Hospital in Gujarat. J Pharma Tech Res. 2015;6(1):29-34.

6. Saurabh MK, Karnani RK. An evaluation of knowledge, attitude and perception about adverse drug reactions and pharmacovigilance among intern doctors at a teaching hospital of Rajasthan. Natl J Physiol Pharm Pharmacol. 2016;6:111-5.

7. Coleman JJ, Pontefract SK. Adverse drug reactions. Clin Med. 2016;16(5):481-5.

8. Gupta R, Sharma D, Malhotra P. Assessment of knowledge, attitude and practice of pharmacovigilance among the undergraduate medical students in a northern Indian tertiary care teaching hospital: an observational study. Int J Pharm Sci Res. 2017;8(6):2654-59.

9. Desai CK, Iyer G, Panchal J, Shah S, Dikshit RK. An evaluation of knowledge, attitude, and practice of adverse drug reaction reporting among prescribers at a tertiary care hospital. Perspect Clin Res. 2011;2:12936.

10. Chatterjee S, Lyle N, Ghosh S. A survey of the knowledge, attitude and practice of adverse drug reaction reporting by clinicians in eastern India. Drug Saf. 2006;29:641-2.

11. Thakuria N, Deka D, Choudhury D, Ahmed N. A cross sectional study to evaluate the knowledge, attitude and practices of healthcare professionals on pharmacovigilance at Silchar medical college and hospital, Assam, India. Int J Basic Clin Pharmacol. 2016;5:1481-9.

12. Korde RA, Radhika MS. A KAP study of pharmacovigilance among junior residents and interns 
of a tertiary care hospital. Int J Basic Clin Pharmacol. 2018;7(11):2178-83.

13. Gupta P, Udupa A. Adverse Drug Reaction Reporting and Pharmacovigilance: Knowledge, Attitudes and Perceptions amongst Resident Doctors. J Pharm Sci Res. 2011;3(2):1064-9.

14. Agarwal M, Ahmed J, Roy V. Knowledge, Attitude, and Practice About Pharmacovigilance Among Healthcare Providers of a Tertiary Care Teaching Hospital in New Delhi (India). MAMC J Med Sci. 2017;3:146-51.

15. Tandon V, Mahajan V, Khajuria V, Gillani Z. Underreporting of adverse drug reactions: A challenge for pharmacovigilance in India. Ind $\mathbf{J}$ Pharmacol. 2015;47(1):65-71.

16. Kalaiselvan V, Prasad T, Bisht A, Singh S, Singh G. Adverse drug reactions reporting culture in Pharmacovigilance programme of India. Ind $\mathrm{J}$ Med Res. 2014;140(4):563-4.

17. Pimpalkhute SA, Jaiswal KM, Sontakke SD, Bajait CS, Gaikwad A. Evaluation of awareness about pharmacovigilance and adverse drug reaction monitoring in resident doctors of a tertiary care teaching hospital. Indian J Med Sci. 2012;66:55-61.

18. Modi PK, Kohli SK, Advani U, Singh H. Evaluation of knowledge, attitude and practice of pharmacovigilance among health care professionals in tertiary care teaching medical college in North India. World J Pharma Res. 2014;3:2691-7.

19. Prakasam A, Nidamanuri A, Kumar S. Knowledge, perception and practice of pharmacovigilance among community pharmacists in South India. Pharm Pract. 2012;10:222-6.

Cite this article as: Shetti SA, Limaye RP. An evaluation of knowledge, attitude and perception about adverse drug reactions and pharmacovigilance among intern doctors in a medical college teaching hospital of Sangli. Int J Basic Clin Pharmacol 2021;10:714-9. 